\title{
A bilinear differential forms approach to parametric structured state-space modelling
}

\author{
P. Rapisarda* and A.C. Antoulas ${ }^{\dagger}$
}

February 9, 2016

\begin{abstract}
We use one-variable Loewner techniques to compute polynomialparametric models for MIMO systems from vector-exponential data gathered at various points in the parameter space. Instrumental in our approach are the connections between vector-exponential modelling via bilinear differential forms and the Loewner framework.
\end{abstract}

Dedicated to the memory of Jan C. Willems- teacher, colleague, friend.

\section{Introduction}

Parametric system identification arises in those areas where system dynamics depends on one or more parameters, e.g. varying geometric or material properties. One approach for its solution is that of [?, ?], based on twovariable rational interpolation techniques and a Loewner matrix associated with the data. In the single-input, single-output case, this approach produces a transfer-function or generalized state-space model depending on one parameter in a higher-order polynomial way.

In this paper we use one-variable Loewner techniques to compute inputstate-output (i/s/o) polynomial-parametric models on the basis of MIMO vector-exponential trajectories produced by a system at various points in the parameter space. Instrumental in our approach are the connections established in [?, ?] between vector-exponential modelling via bilinear differential forms and the Loewner framework. The basic tool in this work is the Loewner matrix and its rank-revealing factorizations, from which a set of state trajectories is computed in a straightforward way. Different factorizations correspond to different state trajectories: we show that by suitably

\footnotetext{
${ }^{*}$ Vision, Learning and Control Group, School of Electronics and Computer Science, University of Southampton, SO17 1BJ Southampton, UK pr3@ecs.soton.ac.uk

${ }^{\dagger}$ Department of Electrical and Computer Engineering, Rice University, Houston, TX 77005, USA, aca@rice.edu; and Jacobs University Bremen, School of Engineering and Science, Bremen, Germany
} 
factorizing the Loewner matrix one can compute also structured polynomialparametric i/s/o models, and we apply this to the case of passive systems.

A few remarks are in order to define the scope of our results. Firstly, no assumptions are made on the parametric dependence of the underlying system, except that at each point in the parameter space where data has been collected the system can be described by a set of linear, constant-coefficient differential equations. Secondly, our choice of model class as that consisting of i/s/o linear, time-invariant models that depend polynomially on a parameter is dictated by purely pragmatic reasons, and does not reflect any intrinsic belief in the nature of the actual dependence on the parameter. Lacking any special insight in the physics of the system it is not reasonable to assume a priori any specific functional dependency on the parameter; moreover, if such detailed physical knowledge is available, there are more suitable approaches than a representation-free one. Finally, it is well-known (see [?, ?]) that functional dependency is not preserved across different representations: for example, an i/o description depending polynomially on a parameter in general does not correspond to an i/s/o polynomially-parametric representation, and vice versa. Our choice of polynomially-dependent parametric i/s/o models is thus motivated purely by practical reasons, namely to identify a "simple" unfalsified (in the sense of [?]) model for the data.

The paper is organized as follows: in section 2 we state the problem, and in section 3 we state the assumptions standing in the rest of the paper. Section 4 contains the main results and is divided in five subsections, dealing with various aspects of our approach. In section 5 we apply our results to the parametric identification of passive systems. In the last section of this paper we discuss our results and their limitations, together with some research directions currently being pursued.

We will be using extensively notions from behavioral system theory, bilinear/quadratic differential forms and the Loewner framework; for a thorough exposition we refer to [?, ?, ?], respectively.

\section{Notation}

The space of $n$ dimensional real (complex) vectors is denoted by $\mathbb{R}^{n}$ (respectively $\mathbb{C}^{n}$ ), and that of $m \times n$ real matrices by $\mathbb{R}^{m \times n}$. $\mathbb{R}^{\bullet \times m}$ denotes the space of real matrices with $m$ columns and an unspecified finite number of rows. Given matrices $A, B \in \mathbb{R}^{\bullet \times m}, \operatorname{col}(A, B)$ denotes the matrix obtained by stacking $A$ over $B$. s The ring of polynomials with real coefficients in the indeterminate $s$ is denoted by $\mathbb{R}[s]$; the ring of two-variable polynomials with real coefficients in the indeterminates $\zeta$ and $\eta$ is denoted by $\mathbb{R}[\zeta, \eta]$. $\mathbb{R}^{r \times q}[s]$ denotes the set of all $r \times q$ matrices with entries in $s$, and $\mathbb{R}^{n \times m}[\zeta, \eta]$ that of $n \times m$ polynomial matrices in $\zeta$ and $\eta$. The set of rational $m \times n$ matrices is denoted by $\mathbb{R}^{m \times n}(s)$.

The set of infinitely differentiable functions from $\mathbb{R}$ to $\mathbb{R}^{q}$ is denoted 
by $\mathfrak{C}^{\infty}\left(\mathbb{R}, \mathbb{R}^{q}\right) \cdot \mathfrak{D}\left(\mathbb{R}, \mathbb{R}^{q}\right)$ is the subset of $\mathfrak{C}^{\infty}\left(\mathbb{R}, \mathbb{R}^{q}\right)$ consisting of compact support functions. Given $\lambda \in \mathbb{C}$, we denote by $e^{\lambda \cdot}$ the exponential function whose value at $t$ is $e^{\lambda t}$, and by $\lambda^{*}$ its complex conjugate.

\section{Problem statement}

We assume that at point $p$ in the parameter space the generating system is controllable, represented in observable image form as

$$
w=M_{p}\left(\frac{d}{d t}\right) \ell,
$$

where $M_{p} \in \mathbb{R}^{\mathrm{w} \times \mathrm{m}}[s]$; we also assume that $w=\left[\begin{array}{l}u \\ y\end{array}\right]$ with $u$ input and $y$ output variables. The input-output partition of the external variables corresponds to a partition

$$
M_{p}(s)=:\left[\begin{array}{c}
U_{p}(s) \\
Y_{p}(s)
\end{array}\right],
$$

where $U_{p} \in \mathbb{R}^{\mathrm{m} \times \mathrm{m}}[s]$ is nonsingular, and $Y_{p} \in \mathbb{R}^{\mathrm{p} \times \mathrm{m}}[s]$.

The data are vector-exponential trajectories at various frequencies and values of the parameter $p$, namely

$$
w_{p_{i}, \lambda_{i, j}}(t)=\overline{w_{p_{i}, \lambda_{i, j}}} e^{\lambda_{i, j} t}, i=1, \ldots, N^{\prime}, j=1, \ldots, N
$$

where $\lambda_{i, j} \in \mathbb{C}$ and $\overline{w_{p_{i}, \lambda_{i, j}}} \in \mathbb{C}^{\mathrm{w}}, i=1, \ldots, N^{\prime}, j=1, \ldots, N$. Since (1) is observable, for every $\overline{w_{p_{i}, \lambda_{i, j}}}$ corresponding to the external trajectory $w_{p_{i}, \lambda_{i, j}}(\cdot)$ there exists a unique vector $\overline{s_{p_{i}, \lambda_{i, j}}} \in \mathbb{C}^{\mathrm{m}}$ such that

$$
\overline{w_{p_{i}, \lambda_{i, j}}}=M_{p_{i}}\left(\lambda_{i, j}\right) \overline{s_{p_{i}, \lambda_{i, j}}} .
$$

Under some assumptions stated in the next section, we want to compute from the data (3) a parametric state-space model

$$
\begin{aligned}
\frac{d}{d t} x & =A(p) x+B(p) u \\
y & =C(p) x+D(p) u
\end{aligned}
$$

where $A(p) \in \mathbb{R}^{n \times n}[p], B(p) \in \mathbb{R}^{n \times \mathrm{m}}[p], C(p) \in \mathbb{R}^{n \times \mathrm{p}}[p]$ and $D(p) \in$ $\mathbb{R}^{\mathrm{m} \times \mathrm{m}}[p]$, with the property that $\left(A\left(p_{i}\right), B\left(p_{i}\right), C\left(p_{i}\right), D\left(p_{i}\right)\right)$ defines an unfalsified state-space model for the data (3); that is, for all $i=1, \ldots, N^{\prime}$ and $k=1, \ldots, N$ there exists a state trajectory $x=x_{i, k}$ satisfying (5) with $\operatorname{col}(u, y)=w_{p_{i}, \lambda_{i, k}}$. Such a model (5) will be called an unfalsified parametric $i / s / o$ model for the data (3). A refinement of such problem consists in requiring also that the transfer function $C(p)\left(s I_{n}-A(p)\right)^{-1} B(p)+D(p)$ is positive-real for all values of $p$. 
In the following we use also a kernel representation of (1):

$$
R_{p}\left(\frac{d}{d t}\right) w=0
$$

where $R_{p} \in \mathbb{R}^{\mathrm{p} \times \mathrm{w}}[s]$ represents the dynamics at the point $p$ in the parameter space. The i/o partition (2) is reflected in the following partition of $R$ :

$$
R_{p}(s)=:\left[Q_{p}(s)-P_{p}(s)\right],
$$

where $P_{p} \in \mathbb{R}^{\mathrm{p} \times \mathrm{p}}[s]$ is nonsingular, and $Q_{p} \in \mathbb{R}^{\mathrm{p} \times \mathrm{m}}[s]$.

\section{Assumptions}

The standing assumptions in the rest of this paper are the following:

1. For each $p_{i}, i=1, \ldots, N^{\prime}$, the first m components of the external variable $w$ are input variables;

2. The transfer function corresponding to each such i/o partition is proper;

3. For each $p_{i}, i=1, \ldots, N^{\prime}$, the McMillan degree of (1) is $n$.

With reference to (2) and (7), and using standard behavioral system theory, it is straightforward to verify that assumptions 1)-3) are equivalent with

$1^{\prime}$. For $i=1, \ldots, N^{\prime}, U_{p_{i}}(s)$ and $P_{p_{i}}(s)$ are nonsingular;

$2^{\prime} . Y_{p_{i}}(s) U_{p_{i}}(s)^{-1}=P_{p_{i}}(s)^{-1} Q_{p_{i}}(s)$ is proper, $i=1, \ldots, N^{\prime}$;

$3^{\prime} . \operatorname{deg}\left(U_{p_{i}}(s)\right)=\operatorname{deg}\left(P_{p_{i}}(s)\right)=n$ for $i=1, \ldots, N^{\prime}$.

We moreover assume that

4. For $i=1, \ldots, N^{\prime}$, the data (3) is sufficiently informative, in the sense that an unfalsified state-space model for the data at point $p_{i}$

$$
\begin{aligned}
\frac{d}{d t} x & =A_{p_{i}} x+B_{p_{i}} u \\
y & =C_{p_{i}} x+D_{p_{i}} u,
\end{aligned}
$$

can be computed from it.

Several different conditions on (3) guarantee that assumption 4) is satisfied; for example, it can be shown that if the following conditions are satisfied:

$4^{\prime} . \quad N>n(n+\mathrm{p}+\mathrm{m})$;

$4^{\prime \prime}$. for $i=1, \ldots, N^{\prime}, \lambda_{i, j} \neq \lambda_{i, k}$ for $j \neq k$,

then a unique model can be computed. 


\section{Parametric state-space modelling}

\section{$4.1 \quad$ Overview}

Our approach is based on the following idea: we first compute from the primal data (3) a set of dual data, i.e. of vector-exponential trajectories generated by the dual system at the value $p_{i}$ of the parameter; crucial in such first step is the concept of mirroring.

Subsequently, for each value of the parameter $p_{i}$ we generate from the primal and dual data a Loewner matrix $\mathbb{L}_{p_{i}} \in \mathbb{C}^{N \times N}$, which we proceed to factorize in a rank-revealing way. From such factorization state trajectories corresponding to the external trajectories (3) for fixed $i$ are readily obtained. Using such state trajectories and the primal data, a model (5) is obtained for $p=p_{i}$ solving a system of linear equations.

We repeat this procedure for $i=1, \ldots, N^{\prime}$. Finally, we compute a parametric state model by combining the pointwise state models thus obtained using standard scalar polynomial-interpolation techniques.

\subsection{Mirroring: computing dual data from primal ones}

We introduce the concept of mirroring, already used in solving metric interpolation problems (see [?, ?, ?]); this is a technique to obtain data generated by the dual system from data obtained from the primal one.

Proposition 1. Let $\mathfrak{B}$ be a controllable linear differential behavior, and let $J \in \mathbb{R}^{\mathrm{w} \times \mathrm{w}}$ represent an involution. Let $w \in \mathfrak{B}, w(t)=\bar{w} e^{\lambda t}$, and let $\bar{v} \in \mathbb{C}^{\mathrm{w}}$ be such that $\bar{v}^{*} \bar{w}=0$. Then $J \bar{v} e^{-\lambda^{*}} \in \mathfrak{B}^{\perp_{J}}$, the dual of $\mathfrak{B}$ with respect to $J$.

Proof. Let $M \in \mathbb{R}^{\mathrm{w} \times \mathrm{m}}[s]$ and $R \in \mathbb{R}^{\mathrm{p} \times \mathrm{w}}[s]$ with $\mathrm{w}=\mathrm{p}+\mathrm{m}$ induce an observable image, respectively left-coprime kernel representation of $\mathfrak{B}$. Since $R(s) M(s)=0_{\mathrm{p} \times \mathrm{m}}$, it follows that for all $\lambda \in \mathbb{C}$, it holds that im $M(\lambda)=$ $\left(\operatorname{im} R(\lambda)^{*}\right)^{\perp}$. It follows that $J \bar{v} e^{-\lambda^{*}} \in \operatorname{im} J R\left(-\frac{d}{d t}\right)^{\top}=\mathfrak{B}^{\perp_{J}}$.

\subsection{The Loewner matrix}

In the following we consider $i$ fixed, but otherwise arbitrarily chosen between 1 and $N^{\prime}$, i.e. we consider the problem of modelling data produced by the system when the parameter $p=p_{i}$. We assume that besides the primal ones, also a set of dual external trajectories

$$
\overline{w_{p_{i}, \mu_{i, k}}^{\prime}} e^{\mu_{i, k} \cdot}, k=1, \ldots, N,
$$

is available (for example through the mirroring technique described in sect. 4.2 ), and we define the Loewner matrix at $p=p_{i}$ by

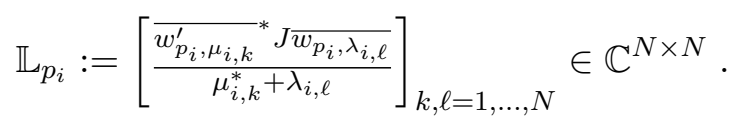


Since $R_{p_{i}}$ induces a minimal kernel representation of a controllable system, it follows that for every $k=1, \ldots, N$ and $i=1, \ldots, N^{\prime}$ there exists a unique vector $\overline{v_{p_{i}, \mu_{i, k}}} \in \mathbb{C}^{\mathrm{P}}$ such that

$$
\overline{w_{p_{i}, \mu_{i, k}}^{\prime}}=J R_{p_{i}}\left(\mu_{i, k}\right)^{\top} \overline{v_{p_{i}, \mu_{i, j}}} .
$$

The first result of this paper is the following.

Theorem 1. Consider the image-and kernel representations (1) and (6). There exists a $\Psi_{p_{i}} \in \mathbb{R}^{\mathrm{p} \times \mathrm{m}}[\zeta, \eta]$ such that

$$
(\zeta+\eta) \Psi_{p_{i}}(\zeta, \eta)=R_{p_{i}}(-\zeta)^{\top} M_{p_{i}}(\eta) \in \mathbb{R}^{\mathrm{p} \times \mathrm{m}}[\zeta, \eta] .
$$

Let $\overline{s_{p_{i}, \lambda_{i, \ell}}}$ and $\overline{v_{p_{i}, \mu_{i, k}}}$ be defined by (4) and (10), respectively. Then

$$
\mathbb{L}_{p_{i}}=-\left[\overline{v_{p_{i}, \mu_{i, k}}} * \Psi_{p_{i}}\left(\mu_{i, k}, \lambda_{i, \ell}\right) \overline{s_{p_{i}, \lambda_{i, \ell}}}\right]_{k, \ell=1, \ldots, N} .
$$

Proof. Observe first that $R_{p_{i}}(s)^{\top} M_{p_{i}}(s)=R_{p_{i}}(s)^{\top} J J M_{p_{i}}(s)=0$, since $R_{p_{i}}(s)$, respectively $M_{p_{i}}(s)$ induce a kernel-, respectively image representation of the same behavior. Now apply the relation between the two-variable representation of a B/QDF and its derivative and Prop. 10.1 p. 1730 of [?] to conclude that there exist $\Psi_{p_{i}} \in \mathbb{R}^{\mathrm{p} \times \mathrm{m}}[\zeta, \eta]$ such that (11) holds.

The second part of the claim follows in a straightforward way from the definition (9) of the Loewner matrix and from equations (4) and (10).

\subsection{From factorizations of $\mathbb{L}_{p_{i}}$ to state-space equations}

We now show that the Loewner matrix $\mathbb{L}_{p_{i}}$ contains enough information to compute state trajectories associated with the external trajectories of the primal- and the dual system at $p=p_{i}$. Recall that given $A \in \mathbb{R}^{n \times m}$, a rankrevealing factorization of $A$ is any factorization $A=U^{\top} V$ with $U \in \mathbb{R}^{r \times n}$, $V \in \mathbb{R}^{r \times m}$ full rank matrices with $r:=\operatorname{rank} A$.

Theorem 2. Denote by $n$ the McMillan degree of im $M_{p_{i}}\left(\frac{d}{d t}\right)$. If assumptions $4^{\prime}, 4^{\prime \prime}$ of sect. 3 holds, and if $N \geq n$, then rank $\mathbb{L}_{p_{i}}=n$.

Moreover, let $\mathbb{L}_{p_{i}}=Z_{p_{i}}^{*} V_{p_{i}}$ be any rank-revealing factorization of the Loewner matrix (9); denote by $V_{p_{i}, k}$, respectively $Z_{p_{i}, \ell}$, the $k$-th, respectively $\ell$-th column of $V_{p_{i}}$, respectively $Z_{p_{i}}$. There exist minimal state representations of $\mathfrak{B}_{p_{i}}:=\operatorname{im} M_{p_{i}}\left(\frac{d}{d t}\right)$, respectively $\mathfrak{B}_{p_{i}}^{\perp_{J}}:=\operatorname{im} J R_{p_{i}}\left(-\frac{d}{d t}\right)^{\top}$, such

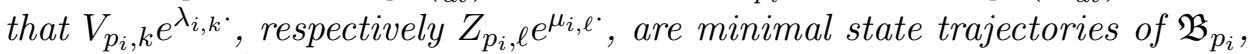
respectively $\mathfrak{B}_{p_{i}}^{\perp J}$.

Proof. To prove the first part of the claim, define $\Psi_{p_{i}}(\zeta, \eta)$ from (11). Conclude from Prop. 10.1 p. 1730 of [?] that there exist state map matrices $Z_{p_{i}} \in \mathbb{R}^{\bullet \times g}[s]$ and $X_{p_{i}} \in \mathbb{R}^{\bullet \times l}[s]$ such that

$$
\Psi_{p_{i}}(\zeta, \eta)=Z_{p_{i}}(\zeta)^{\top} X_{p_{i}}(\eta) .
$$


Conclude that $\mathbb{L}_{p_{i}}=S_{p_{i}}^{*} P_{p_{i}}$, where $S_{p_{i}}$ and $P_{p_{i}}$ are defined by

$$
\begin{aligned}
S_{p_{i}} & :=\left[\begin{array}{lll}
Z_{p_{i}}\left(\mu_{i, 1}^{*}\right) \overline{v_{p_{i}, \mu_{i, 1}}} & \ldots & Z_{p_{i}}\left(\mu_{i, N}^{*}\right) \overline{v_{p_{i}, \mu_{i, N}}}
\end{array}\right] \in \mathbb{C}^{n \times N} \\
P_{p_{i}} & :=\left[\begin{array}{llll}
X_{p_{i}}\left(\lambda_{i, 1}\right) \overline{s_{p_{i}, \lambda_{i, 1}}} & \ldots & X_{p_{i}}\left(\lambda_{i, N}\right) \overline{s_{p_{i}, \lambda_{i, N}}}
\end{array}\right] \in \mathbb{C}^{n \times N} .
\end{aligned}
$$

The rank-revealing factorization of the claim is related to that induced by $S_{p_{i}}$ and $P_{p_{i}}$ by a unitary transformation $T_{p_{i}}$, i.e. $S_{p_{i}}=T_{p_{i}} Z_{p_{i}}$ and $P_{p_{i}}=$ $T_{p_{i}} V_{p_{i}}$, with $T_{p_{i}}^{\top} T_{p_{i}}=I_{r}$. We now prove that assumption 4) implies that $\operatorname{rank}\left(P_{p_{i}}\right)=n$ (equivalently, $\operatorname{rank}\left(V_{p_{i}}\right)=n$ ); the proof is by contradiction.

Assume that $\operatorname{rank}\left(P_{p_{i}}\right)=r<n$; then there exist $\alpha_{k} \in \mathbb{C}, k=1, \ldots, N$, not all zero, such that $P_{p_{i}} \operatorname{col}\left(\alpha_{k}\right)_{k=1, \ldots, N}=0$. Now compute a kernel representation of the most powerful unfalsified model (see sect. XV of [?]) of the subspace of $\mathcal{C}^{\infty}\left(\mathbb{R}, \mathbb{C}^{m}\right)$ spanned by the vector-exponential functions $\overline{s_{p_{i}, \lambda_{i, k}}} e^{\lambda_{i, k}}, k=1, \ldots, N$. Denote by $F_{p_{i}} \in \mathbb{R}^{m \times m}[s]$ such kernel representation. Consider the set of trajectories $w$ described by the equations

$$
\begin{aligned}
w & =M_{p_{i}}\left(\frac{d}{d t}\right) \ell \\
x & =X_{p_{i}}\left(\frac{d}{d t}\right) \ell \\
0 & =F_{p_{i}}\left(\frac{d}{d t}\right) \ell .
\end{aligned}
$$

Such behavior is autonomous (see [?]) since $\operatorname{det}(F) \neq 0$. Moreover, $X_{p_{i}}\left(\frac{d}{d t}\right)$ is a state map for it, since it is a state map for im $M_{p_{i}}\left(\frac{d}{d t}\right)$. Now consider the latent variable trajectory defined by $\hat{\ell}(\cdot):=\sum_{k=1}^{N} \alpha_{k} \overline{s_{i}, \lambda_{i, k}} e^{\lambda_{i, k}}$. Since $P_{p_{i}} \operatorname{col}\left(\alpha_{k}\right)_{k=1, \ldots, N}=0$, the corresponding state trajectory $\hat{x}:=X\left(\frac{d}{d t}\right) \hat{\ell}$ at $t=0$ is zero. It follows from the autonomy of (14) that $\hat{w}:=M_{p_{i}}\left(\frac{d}{d t}\right) \hat{\ell}$ is also zero. Recall that $M_{p_{i}}$ is assumed to be observable (see section 2); it follows then that $\hat{\ell}=0$, which is in contradiction with the assumption that not all $\alpha_{k}$ 's are equal to zero and with assumption $4^{\prime \prime}$. Consequently $P_{p_{i}}$ (equivalently, $V_{p_{i}}$ ) has full rank $n$.

An analogous argument proves that $S_{p_{i}}$ (equivalently, $Z_{p_{i}}$ ) has full rank $n$. Thus the columns of $Z_{p_{i}}$ and those of $V_{p_{i}}$ are directions of state-space trajectories for the dual, respectively primal system.

Remark 1. As mentioned in the proof of Th. 2, rank-revealing factorizations of a given matrix are not unique. Thus by suitably choosing the factorization special state space representations can be obtained; we will apply this later to derive positive-real parametric models.

We now show how to compute an unfalsified i/s/o representation of the data gathered at $p=p_{i}$ from a rank-revealing factorization of $\mathbb{L}_{p_{i}}$. 
Proposition 2. Under assumptions 1) - 3) and $\left.4^{\prime}\right)$, $\left.4^{\prime \prime}\right)$, let $\mathbb{L}_{p_{i}}=Z_{p_{i}}^{*} V_{p_{i}}$ be a rank-revealing factorization of $\mathbb{L}_{p_{i}}$, where $Z_{p_{i}} \in \mathbb{C}^{n \times N}$ and $V_{p_{i}} \in \mathbb{C}^{n \times N}$. Define

$$
\begin{aligned}
\Lambda_{p_{i}} & :=\operatorname{diag}\left(\lambda_{i, k}\right)_{k=1, \ldots, N} \in \mathbb{C}^{N \times N} \\
U_{p_{i}} & :=\left[\begin{array}{lll}
u_{p_{i}, 1} & \ldots & u_{p_{i}, N}
\end{array}\right] \in \mathbb{C}^{\mathrm{m} \times N} \\
Y_{p_{i}} & :=\left[\begin{array}{lll}
y_{p_{i}, 1} & \ldots & y_{p_{i}, N}
\end{array}\right] \in \mathbb{C}^{\mathrm{p} \times N} .
\end{aligned}
$$

There exist $A_{p_{i}} \in \mathbb{C}^{n \times n}, B_{p_{i}} \in \mathbb{R}^{n \times \mathrm{m}}, C_{p_{i}} \in \mathbb{R}^{\mathrm{p} \times n}, D_{p_{i}} \in \mathbb{R}^{\mathrm{m} \times \mathrm{m}}$ such that

$$
\left[\begin{array}{c}
V_{p_{i}} \Lambda_{p_{i}} \\
Y_{p_{i}}
\end{array}\right]=\left[\begin{array}{cc}
A_{p_{i}} & B_{p_{i}} \\
C_{p_{i}} & D_{p_{i}}
\end{array}\right]\left[\begin{array}{c}
V_{p_{i}} \\
U_{p_{i}}
\end{array}\right]
$$

Any $\left(A_{p_{i}}, B_{p_{i}}, C_{p_{i}}, D_{p_{i}}\right)$ satisfying (15) defines a minimal unfalsified statespace model for the data (3).

Proof. The existence of $A_{p_{i}}, B_{p_{i}}, C_{p_{i}}, D_{p_{i}}$ follows from the fact that the columns of the matrix $V_{p_{i}}$ correspond to state-space trajectories of im $M_{p_{i}}\left(\frac{d}{d t}\right)$ (see Th. 2). The second part of the claim follows from the following observation. Denote the $k$-th column of $V_{p_{i}}$ by $V_{p_{i}, k}$, and define $x_{p_{i}, k}(\cdot):=V_{p_{i}, k} e^{\lambda_{i, k}}$, $i=1, \ldots, N$. From (15) we conclude that such trajectory satisfies (8), where $y:=\overline{y_{p_{i}, k}} e^{\lambda_{i, k} \cdot}$ and $u:=\overline{u_{p_{i}, k}} e^{\lambda_{i, k}}$.

\subsection{Polynomial-parametric unfalsified i/s/o models}

Given the set of points $p_{i} \in \mathbb{C}, i=1, \ldots, N^{\prime}$, we consider a set of parametrizing polynomials for $\left\{p_{i}\right\}_{i=1, \ldots, N^{\prime}}$, i.e. $q_{k} \in \mathbb{R}[s], k=1, \ldots, N^{\prime}$ satisfying

$$
q_{k}\left(p_{i}\right)=\delta_{i k}, i, k=1, \ldots, N^{\prime},
$$

with $\delta_{\text {., }}$ being the Kronecker delta; one such set is that consisting of the Lagrange polynomials defined by

$$
q_{k}(p):=\frac{\Pi_{i \neq k}\left(p-p_{i}\right)}{\Pi_{i \neq k}\left(p_{k}-p_{i}\right)} .
$$

If a set of parametrizing polynomials is available, a parametric i/s/o representation is obtained in a straightforward way from the matrices $A_{p_{i}}, B_{p_{i}}$, $C_{p_{i}}, D_{p_{i}}$ satisfying (15).

Theorem 3. Assume conditions 1) - 3) and $\left.4^{\prime}\right)$, $\left.4^{\prime \prime}\right)$, and let $A_{p_{i}}, B_{p_{i}}, C_{p_{i}}$, $D_{p_{i}}, i=1, \ldots, N^{\prime}$, induce unfalsified state-space models for the data (3). Let $\left\{q_{k}(p)\right\}_{k=1, \ldots, N^{\prime}}$ be a family of parametrizing polynomials for $\left\{p_{i}\right\}_{i=1, \ldots, N^{\prime}}$. Define $A \in \mathbb{R}^{n \times n}[p], B \in \mathbb{R}^{n \times \mathrm{m}}[p], C \in \mathbb{R}^{\mathrm{p} \times n}[p]$, and $D \in \mathbb{R}^{\mathrm{p} \times \mathrm{m}}[p]$ by

$$
\begin{aligned}
A(p) & :=\sum_{k=1}^{N^{\prime}} A_{p_{k}} q_{k}(p), B(p):=\sum_{k=1}^{N^{\prime}} B_{p_{k}} q_{k}(p) \\
C(p) & :=\sum_{k=1}^{N^{\prime}} C_{p_{k}} q_{k}(p), D(p):=\sum_{k=1}^{N^{\prime}} D_{p_{k}} q_{k}(p) .
\end{aligned}
$$


Then $(A(p), B(p), C(p), D(p))$ solves the parametric modelling problem.

Proof. Straightforward.

\section{Positive-real parametric identification}

Since we do not commit ourselves earlier on to a specific state representation, and instead compute state sequences using rank-revealing factorizations of the Loewner matrix, we can compute parametric models with special structures and properties. In this section we examine the case of positive-real systems; analogous results hold for the lossless- and adjoint port-Hamiltonian case. Our starting point is a family of representations $\left(A_{p_{i}}, B_{p_{i}}, C_{p_{i}}, D_{p_{i}}\right)$, $i=1, \ldots, N^{\prime}$ (and the dual ones) obtained from solving the equations

$$
\begin{aligned}
& {\left[\begin{array}{c}
V_{p_{i}} \Lambda_{p_{i}} \\
Y_{p_{i}}
\end{array}\right]=\left[\begin{array}{ll}
A_{p_{i}} & B_{p_{i}} \\
C_{p_{i}} & D_{p_{i}}
\end{array}\right]\left[\begin{array}{c}
V_{p_{i}} \\
U_{p_{i}}
\end{array}\right]} \\
& {\left[\begin{array}{c}
Z_{p_{i}} \Lambda_{p_{i}}^{\prime} \\
Y_{p_{i}}^{\prime}
\end{array}\right]=\left[\begin{array}{ll}
-A_{p_{i}}^{\top} & C_{p_{i}}^{\top} \\
-B_{p_{i}}^{\top} & D_{p_{i}}^{\top}
\end{array}\right]\left[\begin{array}{c}
Z_{p_{i}} \\
U_{p_{i}}^{\prime}
\end{array}\right]}
\end{aligned}
$$

Here the $j$-th column of $Y_{p_{i}}$ (respectively $Y_{p_{i}}^{\prime}$ ) is the direction of the output variable of the $j$-th data trajectory of the primal data (respectively dual data), and analogously for $U_{p_{i}}$ and $U_{p_{i}}^{\prime}, i=1, \ldots, N^{\prime}$. $\Lambda$, respectively $\Lambda^{\prime}$, are the diagonal matrices with the primal-, respectively dual-, interpolation points.

We want to compute a parameter-depending realization (5), whose associated transfer function is positive-real for all values of $p$. In the following, we call a realization $(A, B, C, D)$ of a positive-real transfer function $H(s)$ unit-storage if $K=I$ is a solution of the positive-real lemma

$$
\left[\begin{array}{cc}
-K & 0 \\
0 & I
\end{array}\right]\left[\begin{array}{ll}
A & B \\
C & D
\end{array}\right]+\left[\begin{array}{ll}
A^{\top} & C^{\top} \\
B^{\top} & D^{\top}
\end{array}\right]\left[\begin{array}{cc}
-K & 0 \\
0 & I
\end{array}\right] \geq 0
$$

Proposition 3. Every positive-real transfer function $H(s)$ admits a unitstorage realization.

Proof. Let $(A, B, C, D)$ be any realization of $H(s)$. Apply the positive-real Lemma to conclude that there exists $K=K^{\top}>0$ such that (20) holds. Factorize $K=S^{\top} S$ with $S$ nonsingular, and define $A^{\prime}:=S^{-1} A S, B^{\prime}:=S B$, $C^{\prime}:=C S^{-1}, D^{\prime}:=D$. It is straightforward to check that $\left(A^{\prime}, B^{\prime}, C^{\prime}, D^{\prime}\right)$ is unit-storage.

Different rank-revealing factorizations of the Loewner matrix $\mathbb{L}_{p_{i}}$ produce different state trajectories; and such directions in their turn correspond through (19) to different state representations $\left(A_{p_{i}}, B_{p_{i}}, C_{p_{i}}, D_{p_{i}}\right)$. We now characterize those factorizations of $\mathbb{L}_{p_{i}}$ that yield unit-storage realizations.

We begin with a data-based test for positive-realness. 
Proposition 4. Assume that for $i=1, \ldots, N^{\prime}$ the number $N$ of data is such that $N \gg n+\mathrm{m}$, and that for any rank-revealing factorization $\mathbb{L}_{p_{i}}=Z_{p_{i}}^{*} V_{p_{i}}$ it holds that $\mathrm{rank}\left[\begin{array}{c}V_{p_{i}} \\ U_{p_{i}}\end{array}\right]=\operatorname{rank}\left[\begin{array}{c}Z_{p_{i}} \\ U_{p_{i}}^{\prime}\end{array}\right]=n+\mathrm{m}$. The following statements are equivalent:

1. The realization $\left(A_{p_{i}}, B_{p_{i}}, C_{p_{i}}, D_{p_{i}}\right)$ is positive-real;

2. The realization $\left(-A_{p_{i}}^{\top}, C_{p_{i}}^{\top}, B_{p_{i}}^{\top}, D_{p_{i}}^{\top}\right)$ is positive-real;

3. There exists $K_{p_{i}}=K_{p_{i}}^{\top}>0$ such that

$$
Y_{p_{i}}^{*} U_{p_{i}}+U_{p_{i}}^{*} Y_{p_{i}} \geq V_{p_{i}}^{*} K_{p_{i}} V_{p_{i}} \Lambda_{p_{i}}+\Lambda_{p_{i}}^{*} V_{p_{i}}^{*} K_{p_{i}} V_{p_{i}}
$$

4. There exists $K_{p_{i}}^{\prime}=K_{p_{i}}^{\prime \top}>0$ such that

$$
Y_{p_{i}}^{\prime *} U_{p_{i}}^{\prime}+U_{p_{i}}^{\prime *} Y_{p_{i}} \geq-\left(Z_{p_{i}}^{*} K_{p_{i}}^{\prime} Z_{p_{i}} \Lambda_{p_{i}}^{\prime}+\Lambda_{p_{i}}^{\prime *} Z_{p_{i}}^{*} K_{p_{i}}^{\prime} Z_{p_{i}}\right)
$$

Proof. The equivalence between statements 1 . and 2 . follows in a straightforward way from the definition of positive-real function. To prove the equivalence of 1 . and 3., use the assumption rank $\left[\begin{array}{c}V_{p_{i}} \\ U_{p_{i}}\end{array}\right]=n+\mathrm{m}$ and multiply (20) on the left by $\left[\begin{array}{c}V_{p_{i}} \\ U_{p_{i}}\end{array}\right]^{*}$ and on the right by $\left[\begin{array}{c}V_{p_{i}} \\ U_{p_{i}}\end{array}\right]$ to obtain (3).

A similar argument proves the equivalence of 2 . and 4 .

The following consequence of Prop. 4 gives a characterization of unitstorage realizations in terms of data.

Corollary 1. Under the assumptions and notation of Prop. 4, the following statements are equivalent:

1. The realization $\left(A_{p_{i}}, B_{p_{i}}, C_{p_{i}}, D_{p_{i}}\right)$ is positive-real and unit-storage;

2. The realization $\left(-A_{p_{i}}^{\top}, C_{p_{i}}^{\top}, B_{p_{i}}^{\top}, D_{p_{i}}^{\top}\right)$ is positive-real and unit-storage;

3. The following LMI holds:

$$
\left[\begin{array}{cc}
-I & 0 \\
0 & I
\end{array}\right]\left[\begin{array}{ll}
A_{p_{i}} & B_{p_{i}} \\
C_{p_{i}} & D_{p_{i}}
\end{array}\right]+\left[\begin{array}{cc}
A_{p_{i}}^{\top} & C_{p_{i}}^{\top} \\
B_{p_{i}}^{\top} & D_{p_{i}}^{\top}
\end{array}\right]\left[\begin{array}{cc}
-I & 0 \\
0 & I
\end{array}\right] \geq 0
$$

4. The following LMI holds:

$$
Y_{p_{i}}^{*} U_{p_{i}}+U_{p_{i}}^{*} Y_{p_{i}} \geq V_{p_{i}}^{*} V_{p_{i}} \Lambda_{p_{i}}+\Lambda_{p_{i}}^{*} V_{p_{i}}^{*} V_{p_{i}}
$$

5. The following LMI holds:

$$
Y_{p_{i}}^{\prime *} U_{p_{i}}^{\prime}+U_{p_{i}}^{\prime *} Y_{p_{i}}^{\prime} \geq-\left(Z_{p_{i}}^{*} Z_{p_{i}} \Lambda_{p_{i}}^{\prime}+\Lambda_{p_{i}}^{*} Z_{p_{i}}^{*} Z_{p_{i}}\right)
$$


Proof. It follows in a straightforward manner from Prop. 4.

Remark 2. Given the crucial role of unit-storage realizations in what follows, it is of interest to develop computationally efficient and numerically accurate algorithms to perform the computation of factorizations $\mathbb{L}_{p_{i}}=Z_{p_{i}}^{*} V_{p_{i}}$ resulting through (19) in unit-storage realizations. This is a matter for future research; we only sketch a couple of possible solutions here. We could factor $\mathbb{L}_{p_{i}}$ arbitrarily, and then solve for $K_{p_{i}}$ an LMI like that in statement (3) or (4) of Prop. 4. Factorizing $K_{p_{i}}=S_{p_{i}}^{\top} S_{p_{i}}$, defining $V_{p_{i}}^{\prime}:=S_{p_{i}} V_{p_{i}}$ and solving the equations (19) yields a unit-storage realization. Alternatively, one can first compute matrices $\left(A_{p_{i}}, B_{p_{i}}, C_{p_{i}}, D_{p_{i}}\right)$ satisfying (19), solve the positivereal lemma for some $K_{p_{i}}$, and then compute from a factorization of $K_{p_{i}}$ the state-space transformation $S_{p_{i}}$ yielding a unit-storage realization.

Having computed a unit-storage realization of an interpolant for the data at $p=p_{i}, i=1, \ldots, N^{\prime}$, it follows from the inequality in statement (3) of Corollary 1 that any convex combination of the system matrices $\left[\begin{array}{cc}A_{p_{i}} & B_{p_{i}} \\ C_{p_{i}} & D_{p_{i}}\end{array}\right]$ also satisfies the inequality, and is consequently unit-storage positive-real. Thus if a family of parametrizing polynomials exists providing a "convex combination" of such models, then it results in a positive-real, and unitstorage, parametric interpolant.

Proposition 5. Let the data (3) be given. Assume conditions 1) - 3) and $\left.\left.4^{\prime}\right), 4^{\prime \prime}\right)$ of sect. 3 , and that for any rank-revealing factorization $\mathbb{L}_{p_{i}}=Z_{p_{i}}^{*} V_{p_{i}}$ it holds that rank $\left[\begin{array}{c}V_{p_{i}} \\ U_{p_{i}}\end{array}\right]=\operatorname{rank}\left[\begin{array}{c}Z_{p_{i}} \\ U_{p_{i}}^{\prime}\end{array}\right]=n+\mathrm{m}$. Assume also that any of the statements of Prop. 4 holds.

For $i=1, \ldots, N^{\prime}$, there exists an unfalsified unit-storage positive-real realization (8). If there exist $\alpha_{i} \in \mathbb{R}[p], i=1, \ldots, N^{\prime}$ such that

1. $\alpha_{i}\left(p_{j}\right)=\delta_{i j}$, the Kronecker delta function;

2. $\alpha_{i}(p) \geq 0$,

then

$$
\begin{aligned}
\frac{d}{d t} x & =\underbrace{\left(\sum_{i=1}^{N^{\prime}} \alpha_{i}(p) A_{p_{i}}\right)}_{=: A(p)} x+\underbrace{\left(\sum_{i=1}^{N^{\prime}} \alpha_{i}(p) B_{p_{i}}\right)}_{=: B(p)} u \\
y & =\underbrace{\left(\sum_{i=1}^{N^{\prime}} \alpha_{i}(p) C_{p_{i}}\right)}_{=: C(p)} x+\underbrace{\left(\sum_{i=1}^{N^{\prime}} \alpha_{i}(p) D_{p_{i}}\right)}_{=: D(p)} u,
\end{aligned}
$$

is an unfalsified unit-storage positive-real realization for (3). 
Proof. That unfalsified unit-storage realizations exist for each $p_{i}$ follows from Prop. 3. To prove the second part of the claim, let $\left\{\alpha_{i}(\cdot)\right\}_{i=1, \ldots, k}$ be any set of polynomials satisfying the conditions (1) - (2); then

$$
\left[\begin{array}{cc}
-I & 0 \\
0 & I
\end{array}\right]\left[\begin{array}{ll}
A_{p_{i}} & B_{p_{i}} \\
C_{p_{i}} & D_{p_{i}}
\end{array}\right]+\left[\begin{array}{cc}
A_{p_{i}}^{\top} & C_{p_{i}}^{\top} \\
B_{p_{i}}^{\top} & D_{p_{i}}^{\top}
\end{array}\right]\left[\begin{array}{cc}
-I & 0 \\
0 & I
\end{array}\right] \geq 0
$$

if and only if for all $p$

$$
\left[\begin{array}{cc}
-I & 0 \\
0 & I
\end{array}\right] \alpha_{i}(p)\left[\begin{array}{ll}
A_{p_{i}} & B_{p_{i}} \\
C_{p_{i}} & D_{p_{i}}
\end{array}\right]+\alpha_{i}(p)\left[\begin{array}{cc}
A_{p_{i}}^{\top} & C_{p_{i}}^{\top} \\
B_{p_{i}}^{\top} & D_{p_{i}}^{\top}
\end{array}\right]\left[\begin{array}{cc}
-I & 0 \\
0 & I
\end{array}\right] \geq 0
$$

which implies that for all $p$

$$
\left[\begin{array}{cc}
-I & 0 \\
0 & I
\end{array}\right] \sum_{i=1}^{k} \alpha_{i}(p)\left[\begin{array}{cc}
A_{p_{i}} & B_{p_{i}} \\
C_{p_{i}} & D_{p_{i}}
\end{array}\right]+\sum_{i=1}^{k} \alpha_{i}(p)\left[\begin{array}{cc}
A_{p_{i}}^{\top} & C_{p_{i}}^{\top} \\
B_{p_{i}}^{\top} & D_{p_{i}}^{\dagger}
\end{array}\right]\left[\begin{array}{cc}
-I & 0 \\
0 & I
\end{array}\right] \geq 0
$$

which is equivalent to

$$
\left[\begin{array}{cc}
-I & 0 \\
0 & I
\end{array}\right]\left[\begin{array}{ll}
A(p) & B(p) \\
C(p) & D(p)
\end{array}\right]+\left[\begin{array}{ll}
A(p)^{\top} & C(p)^{\top} \\
B(p)^{\top} & D(p)^{\top}
\end{array}\right]\left[\begin{array}{cc}
-I & 0 \\
0 & I
\end{array}\right] \geq 0
$$

This proves the claim.

Remark 3. It is a matter of immediate verification to check that the polynomials $\alpha_{i}(p)$ satisfying conditions 1$)-2$ ) of Prop. 5 can be chosen as $\alpha_{i}(p)=q_{i}(p)^{2}$, where $q_{i}$ is the $i$-th Lagrange polynomial defined in (17). In practical applications it may be required of the $\alpha_{i}(\cdot)$ that they are nonnegative only in some subset of the parameter space, for example corresponding to physically meaningful realizations. Also in the multi-parameter case, the computation of such polynomials $\alpha_{i}(\cdot)$ is a multivariate nonnegative interpolation problem, which in the case $d$ (number of parameters) equal to 1 or 2 is solvable also when the nonnegativity condition is satisfied only on a restriction of the whole parameter space (see [?]).

Relevant research questions regarding this issue are the determination of efficient, numerically sound computational procedures to produce polynomials $\alpha_{i}(\cdot)$ satisfying the conditions; and the refinement of such algorithms to include for example "averaging" of the effects of the parameters (e.g. by augmenting conditions 1$)-2$ ) of Prop. 5 on the $\alpha_{i}(\cdot)$ with $\sum_{i=1}^{N^{\prime}} \alpha_{i}(p)=1$ in the relevant area of the parameter space).

Remark 4. Using the results in Th. 1, and Prop.s 1 and 4 of [?], a methodology analogous to that illustrated in this section can be followed to provide lossless- and self-adjoint Hamiltonian parametric realizations. For reasons of space we do not enter into such details. 


\section{Conclusions}

We presented an approach to the identification of polynomial-parametric i/s/o models based on rank-revealing factorization of Loewner matrices. Our approach allows structured parametric representations to be computed in a straightforward way from the data; we have illustrated the case of positivereal systems, but analogous results also hold for lossless- and self-adjoint port-Hamiltonian systems.

Our results fall short of a completely satisfactory answer to the parametric structured interpolation problem: our procedure imposes a polynomialparametric structure on an identified set of models (see the definition of parametrizing polynomials in (16), and the definition of the $\alpha_{i}(\cdot)$ in Prop. 5). The approach of [?, ?] lets the data speak for themselves instead, by identifying simultaneously the model and its (polynomial) dependence on one parameter. The most pressing direction for further research is to encompass such generality in the BDF framework. Another important research question is the application of the BDF approach to (structured) parametric model order reduction problems (see also [?]). 